\title{
Evolutionary Coalitional Games
}

\author{
Tadeusz Płatkowski
}

Published online: 21 February 2015

(c) The Author(s) 2015. This article is published with open access at Springerlink.com

\begin{abstract}
We introduce the concept of evolutionary coalitional games played in a large population. The members of the population play a strategy chosen from a finite set and interact in randomly formed coalitions. The interactions are described by a multiplayer strategic game. Each coalition generates a total utility, identified with the value of the coalition, and equal to the sum of the payoffs of its all members from the multiplayer game. The total utility is distributed among the coalition members, proportionally to their Shapley values. Evolution of the whole population is governed by the replicator equations. Polymorphic stationary states of the population are studied for various types of the multiplayer social dilemma games. It is argued that application of coalitional game theory solution concepts to social dilemma models of evolutionary game theory can foster cooperation in the long run.
\end{abstract}

Keywords Population dynamics · Multiplayer games $\cdot$ Shapley value · Replicator dynamics · Social dilemmas

\section{Introduction}

In the classical game theory three types of games: strategic, coalitional, and evolutionary games, are often introduced as separate topics, which among others reflects the fact that they correspond to various solution concepts and different levels of description of a set of agents. While the strategic games provide a description of individual interactions between the agents on a microscopic level, the coalitional games describe the behavior of coalitions of the agents, i.e., form an intermediate, mesoscopic level, whereas the evolutionary games describe the time behavior of large systems of interacting agents on the macroscopic, population level. Moreover, the most popular solution concept of a strategic game is based on the Nash equilibrium [16]; the solution concepts of a coalitional game with transferable utilities, cf. e.g., [9], are based on the relevant partition of the total utility of a coalition, whereas the

T. Płatkowski $(\bowtie)$

Faculty of Mathematics, Informatics, and Mechanics, University of Warsaw, Warsaw, Poland

e-mail: tplatk@mimuw.edu.pl 
solution of an evolutionary game can be thought of as an asymptotically stable point of replicator equations, determining the frequencies of different behavioral types in equilibrium $[4,20,21]$.

We propose a framework that combines these three types of description into one mathematical model. We consider a large population of agents who play a multiplayer strategic game in randomly formed coalitions; however, their final individual utilities are determined from their contributions to the total value of the coalition, as derived from a chosen type of a coalitional solution. In this note, we choose the Shapley value. To investigate the evolution of the frequency of different behavioral types (strategies), we study the dynamics of the population in the evolutionary setting described by the replicator equations. We investigate the temporal evolution of the fractions of available strategies as a function of the payoffs from the interactions (described by a multiplayer game) within the groups.

This general framework is applied to study the evolution of a population of players whose individual interactions are described by the multiperson social dilemma games, in particular by the Prisoner's Dilemma (PD) game and the Public Goods (PG) games.

In the evolutionary game theory and in the social sciences, the PD game is studied as a paradigm for the evolution of cooperation, cf. e.g., [4,10,21]. In populations of individuals, randomly matched to play the PD game or its multiplayer versions, such as the PG games, the evolutionary theory based on the replicator equations shows that the fraction of cooperators tends to zero in the subsequent generations. As we show, this is in general not the case in the proposed model. The population may evolve to an asymptotic stationary state with full or partial cooperation. Moreover, the stationary state corresponding to full defection is never globally attracting.

Thus, apart of its own interest as the formalism which embraces into one model two different types of games, the strategic games and the coalitional games, the model shows that application of the solution concepts of the coalitional game theory to models of social dilemmas in the framework of evolutionary game theory can foster cooperation in the long run.

The next section presents the general model with arbitrary number of strategies and arbitrary order of the multiperson game. In Sect. 3, we apply the general theory to populations in which the coalitions of cardinality three are formed, and the agents play a three-player PD game or a PG game with a threshold. In the last section, we conclude and discuss some directions of future work and generalizations. In "Appendix", we provide analytical conditions sufficient to foster cooperation in redistributed general two-person games: PD, coordination, and anti-coordination games.

\section{The Model}

We consider a large population of objects, agents, that at each instant of time are randomly matched into finite groups of the same cardinality $n \geq 2$. The agents are wired to one of $s$ types of strategies. The strategy played is the only feature that identifies the agent. Thus, the agents using the same strategy are indistinguishable.

In each group, the agents interact. The interactions are described by a given $n$-player strategic game

$$
<N, A,\left(u_{i}\right)_{i \in N}>
$$

where $N=\{1,2, \ldots, n\}$, the utility $u_{i}$ of the $i$ th agent depends on its strategy and the strategies played by the $n-1$ opponents taken from a finite set $A$ of $s=|A|$ available strategies and is identified with the payoff of $i$ th player from the $n$-player game. 
The payoffs of all $n$ players are collected together and redistributed between them by an external party, using a prescribed procedure. In order to specify the procedure, we shall use formalism of coalitional games.

According to the general prescription of the coalitional game theory, it is assumed that the agents in the coalitions agree to redistribute the total wealth of the coalition (i.e., the sum of the payoffs of the individuals from the underlining $n$-person strategic game) among themselves proportionally to their strength in the coalitions, measured according to a welldefined solution concept of the coalition game theory. Optionally, one can assume that an external party enforces the redistribution of the total wealth of the coalition according to this solution. It is assumed then that the intervention of the external party has some constraints, in particular it can not redistribute the total income arbitrarily, for example, promoting arbitrarily certain behaviors at the costs of other ones.

Any subset of $N$ will be referred to as a coalition. The whole group of $n$ agents forms a grand coalition. The sum $v(N)$ of the individual utilities of the agents in the grand coalition (the value of the coalition of $n$ agents) is redistributed between its members according to a prescribed procedure, a coalitional solution. In this paper as a coalitional solution, we choose the Shapley value (see Sect. 4 for a discussion of other solution concepts). The Shapley value (we use the abbreviation $\mathrm{SV}$ ) is one of the most popular and widely accepted solution concepts of the coalitional game theory and has been derived axiomatically in [18]. SV assigns to each player in the grand coalition his share in the total wealth of the coalition. To determine the SV's, we need to define the values of all coalitions of lower cardinalities. We assume that the value of the empty coalition and of each singleton coalition is zero (the agents do not generate any utility acting alone). The value $v(K)$ of a coalition $K$ of cardinality $k \in\{2, \ldots, n\}$ is defined as the sum of the individual utilities of the agents in $K$ from a prescribed $k$-player strategic game which would be played once such coalition would have been formed. In such a way, we define the values $v(K)$ of all coalitions $K \subset N$ and the coalitional game in the characteristic form

$$
<N, v(K)>\text {. }
$$

The redistribution of the total wealth of a coalition according to the (strategy dependent) SV's of the players will be called SV redistribution. The SV of the player $i \in N$ in the grand coalition reads $[9,18]$ :

$$
S V_{i}(v)=\frac{1}{n !} \sum_{K: i \in K}(|K|-1) !(n-|K|) ! \Delta_{i}(K) \quad \forall i=1, \ldots, n,
$$

where $\Delta_{i}(K):=v(K)-v(K \backslash\{i\})$ is the marginal contribution of the player $i \in K$ to the coalition $K$. The intuition behind the formula defining the SV is that an agent $i$ brings to the set $K \backslash\{i\}$ of $|K|-1$ agents who already entered the coalition his marginal contribution $v(K)-v(K \backslash\{i\})$. Assuming that the agents enter the coalitions in random order and that all orders have the same probability, the $\mathrm{SV}$ of an agent $i$ is the average of all his marginal contributions. Besides its intuitive appeal, the SV has also firm axiomatic foundations, broadly discussed and extended in literature, cf. e.g., [7], and literature cited therein.

Formally, the redistribution of the total wealth leads to another strategic game, with the same sets of available strategies and with the redistributed individual payoffs. For brevity, this new game will be referred to as the SV game.

To study the evolution of the population, we use the framework of the evolutionary game theory. Applications of the evolutionary game theory to social systems have been discussed e.g., in $[4,17,21]$, where various types of imitative dynamics, describing microscopic interac- 
tions between individuals have been shown to lead to the replicator equations. Optionally, the redistributed individual payoffs of the players can be used to generate copies of the players (i.e., the players with the same strategies) in quantities proportional to their redistributed payoffs. The copies of all players in the population from all grand coalitions are mixed together and form a new generation-the population in the next time step. In the general framework of the evolutionary game theory, this process is approximated by the system of replicator equations, which describe the evolution of the strategy frequencies in the population:

$$
\frac{\mathrm{d} q_{i}(t)}{\mathrm{d} t}=q_{i}\left(U_{i}-\bar{U}\right)
$$

where $q_{i}$ is the frequency of strategy $i \in A$ in the population, $U_{i}$ is the SV redistributed utility of a player who uses strategy $i, \bar{U}=\sum_{k=1}^{k=s} q_{k} U_{k}$ is the SV redistributed utility of a randomly chosen player, and all functions on the rhs are taken at time $t$.

In the next section, we study the temporal evolution of the population in the long run. In particular, we investigate the evolution of the frequencies of available strategies and the existence and stability of polymorphic stationary states of the population in various types of multiplayer games. In particular, we study the PG games with thresholds and the PD games, in the case when three-player coalitions are formed.

\section{Three-Player Coalitions}

We apply the general formalism to three-player coalitions $(n=3)$ in which each player can choose between two strategies, $C$, and $D$, and the underlining three-person strategic game has the payoff matrix

\begin{tabular}{c|ccc} 
& $C C$ & $C D$ & $D D$ \\
\hline$C$ & $R^{\prime}$ & $S^{\prime}$ & $S^{\prime \prime}$ \\
$D$ & $T^{\prime \prime}$ & $T^{\prime}$ & $P^{\prime}$
\end{tabular}

Due to applications of the general theory to social dilemma games, strategy $\mathrm{C}$ will be referred to as cooperation and D as defection. The entries $R^{\prime}, S^{\prime}, S^{\prime \prime}\left(T^{\prime \prime}, T^{\prime}, P^{\prime}\right)$ in the first (second) row of the payoff matrix are the payoffs of a cooperator (defector), respectively, against two cooperators, a cooperator and a defector, and against two defectors.

According to the general model defined in the previous section, a three-player (grand) coalition generates a total utility (value), defined as the sum of the payoffs of all players from the three-player strategic game (3). In order to calculate the Shapley values of the players in the grand coalition, we assume that the value of a two-player coalition is a sum of the payoffs of both players from a two-player symmetric game with the payoff matrix

$$
\begin{array}{c|cc} 
& C & D \\
\hline C & R & S \\
D & T & P
\end{array}
$$

(we use the standard notation from the two-player PD game). For example, $v(\{C, D\})=$ $T+S$. For simplicity of notation, the payoff matrix (4) will be identified with the vector $[R, S, T, P]$.

The values $v(N)$ of all types of three-player coalitions and the Shapley values [calculated from (1)] of the cooperators $\left(S V_{C}\right)$ and defectors $\left(S V_{D}\right)$ in these coalitions are presented in the table: 


\begin{tabular}{r|ccc}
$N$ & $v(N)$ & $S V_{C}$ & $S V_{D}$ \\
\hline$\{C C C\}$ & $3 R^{\prime}$ & $R^{\prime}$ & - \\
$\{C C D\}$ & $T^{\prime \prime}+2 S^{\prime}$ & $F_{1}$ & $F_{2}$ \\
$\{C D D\}$ & $2 T^{\prime}+S^{\prime \prime}$ & $F_{3}$ & $F_{4}$ \\
$\{D D D\}$ & $3 P^{\prime}$ & - & $P^{\prime}$
\end{tabular}

where

$$
\begin{aligned}
& F_{1}=\frac{1}{3}\left[2 S^{\prime}+T^{\prime \prime}+R-(T+S) / 2\right], \quad F_{2}=\frac{1}{3}\left[2 S^{\prime}+T^{\prime \prime}-2 R+T+S\right], \\
& F_{3}=\frac{1}{3}\left[S^{\prime \prime}+2 T^{\prime}-2 P+T+S\right], \quad F_{4}=\frac{1}{3}\left[S^{\prime \prime}+2 T^{\prime}+P-(T+S) / 2\right] .
\end{aligned}
$$

After redistribution of the value of the three-person coalition between its members proportionally to their SV's, the initial game (3) is replaced by the SV game

\begin{tabular}{c|ccc} 
& $C C$ & $C D$ & $D D$ \\
\hline$C$ & $R^{\prime}$ & $F_{1}$ & $F_{3}$ \\
$D$ & $F_{2}$ & $F_{4}$ & $P^{\prime}$
\end{tabular}

According to the general model, the copies of all players in the population are generated in quantities proportional to their final payoffs and are randomly mixed together to form the next generation of the population. Let us denote $q=q(t)$-the frequency of $C$-strategy in the whole population at time $t$. The assumption of a very large (formally infinite) system enables to calculate the probability of all types of three-player interactions in the population, for example, the probability that at time $t$ a player interacts with a pair $\{C, D\}$ is $2 q(1-q)$.

In the evolutionary scenario, we assume that the evolution of the state of the population (identified here with the fraction of cooperators) is governed by the replicator equations (2). In the case of two strategies, they reduce to one equation

$$
\frac{\mathrm{d} q(t)}{\mathrm{d} t}=q\left(U_{C}-\bar{U}\right)=q(1-q)\left(U_{C}-U_{D}\right)=q(1-q) W(q),
$$

where $q$ is the frequency of $C$-strategy, $U_{C}$ and $U_{D}$, derived from (8), are, respectively, the mean payoffs of a cooperator and defector, randomly chosen from the whole population, $\bar{U}=q U_{C}+(1-q) U_{D}$, and

$$
W(q):=A q^{2}+B q+C=0,
$$

$A=R^{\prime}-P^{\prime}+2\left(F_{4}-F_{1}\right)+F_{3}-F_{2}, \quad B=2\left(F_{1}-F_{3}-F_{4}\right)+2 P^{\prime}, \quad C=F_{3}-P^{\prime}$.

Two stationary states of (9): $q=0$, and $q=1$, correspond, respectively, to full defection and full cooperation. The other (called mixed below) stationary states (if they exist) are identified with zeros of $W(q)$.

It is easy to formulate sufficient conditions for the existence of one or two mixed stationary states and their stability for the general three-player game, e.g., as the conditions for the signs of the coefficients $A, B, C$ in (9). We omit the corresponding general expressions and discuss interesting examples of social dilemma games. We present results for the three-player PG games with a threshold and for the PD game. In particular, we study the mixed stationary states of the evolving population in the formalism of the replicator equations (9) for such games.

In the PG game each player receives a unit of a good and contributes (action $C$ ) or does not contribute (action $D$ ) with the unit to the common pool. The amount in the common pool is multiplied by $r>1$ and divided equally among all players, but only if the number of cooperators is at least $M, 1 \leq M \leq 3$. Otherwise the contributions are lost and $C$-players 
receive 0 , whereas $D$-players keep their initial endowments as their final payoffs. The case $M=1$ will be referred to as the PG game (and is a particular case of a general three-person PD game studied below), the cases $M=2$ and 3 will be called the PG games with the relevant threshold $M$.

First, we define the relevant three-player PG games and discuss the equilibrium states of the population evolving according to the replicator equations without the redistribution procedure.

\section{Example 1 (three-player PG games)}

1a. Threshold $M=1$. The payoff matrix of the three-player PG game reads

$$
\begin{array}{c|ccc} 
& C C & C D & D D \\
\hline C & r & 2 r / 3 & r / 3 \\
D & 2 r / 3+1 & r / 3+1 & 1
\end{array}
$$

with $r \in(1,3)$. Note that strategy $\mathrm{D}$ strictly dominates strategy $C$; therefore, the replicator dynamics based on the PG game without redistribution gives $D$ (corresponding to $q=0$ ) as the asymptotic equilibrium state. The game (11) is a particular case of the general three-person PD game, discussed in example 2 below.

1b. Threshold $M=2$. The payoff matrix of this $\mathrm{PG}$ game reads

\begin{tabular}{c|ccc} 
& $C C$ & $C D$ & $D D$ \\
\hline$C$ & $r$ & $2 r / 3$ & 0 \\
$D$ & $2 r / 3+1$ & 1 & 1
\end{tabular}

with $r \in(1,3)$. This game can be seen as an "intermediate" social dilemma game between the PD game 1a and the Stag Hunt game 1c below. Without redistribution of the total wealth the replicator equations for the game $1 \mathrm{~b}, \frac{\mathrm{d} q(t)}{\mathrm{d} t}=q(1-q)\left(-q^{2} r+4 q r / 3-1\right)$ imply that for $r<9 / 4$ the point $q=0$, corresponding to total defection, is globally stable in $[0,1)$. For $r=9 / 4$, the equilibrium $q=\frac{2}{3}$ is a one-dimensional saddle point, whereas for $3>r>9 / 4$, there exist two equilibrium points $q_{1,2}=\frac{2}{3} \mp \frac{\sqrt{\Delta}}{2 r} \in(0,1), q_{1}<$ $q_{2}, \Delta=\frac{16 r^{2}}{9}-4 r$, corresponding to partial cooperation in the long run. $q_{1}$ is a repeller; $q_{2}$ is an attractor of the replicator dynamics. For $r \geq 3$, the point $q_{1}$ is an unique mixed equilibrium, a repeller, and $q_{1}$ decreases when $r$ increases.

1c. Threshold $M=3$. The payoff matrix of the $\mathrm{PG}$ game

$$
\begin{array}{c|ccc} 
& C C & C D & D D \\
\hline C & r & 0 & 0 \\
D & 1 & 1 & 1
\end{array}
$$

defines a three-person Stag Hunt game with the payoff $r>1$ for hunting the stag and 1 for hunting the hare for each player. Without redistribution of the total wealth, the replicator equation for the game $1 \mathrm{c}, \frac{\mathrm{d} q(t)}{\mathrm{d} t}=q(1-q)\left(r q^{2}-1\right)$, implies that for all $r>1$, the point $q_{2}=\frac{1}{\sqrt{r}}$ is a repeller in $(0,1)$. As we shall see, in all above PG games, the redistribution of the total wealth proportionally to the Shapley values of the players foster cooperation in the long run.

First, we carry out the redistribution procedure for the game 1a. The values of threeplayers coalitions are sums of the individual payoffs of their members: $v(\{C, C, C\})=$ $3 r, v(\{C, C, D\})=2 r+1, v(\{C, D, D\})=r+2, v(\{D, D, D\})=3$. In order to define 
the relevant coalitional game, we have to determine the values of all two-player coalitions. We assume that the value of a two-player coalition is the sum of the payoffs of its both members from a two-player game. We choose three different types of two-player games, each one being a particular case of an $N$-person social dilemma for $N=2$.

A1. Two-player PG game, in which the total amount of the common pool is multiplied by $d \in(1,2)$ and distributed between both players (in fact equally for such two-player coalitions). This two-player PG game is a particular case of the general PD game with the payoff matrix (4) with $[R, S, T, P]=[d, d / 2, d / 2+1,1]$.

A2. Two-player PD game in the benefit cost formulation, with the payoff matrix $[R, S, T, P]=[b-c,-c, b, 0], b>c$.

A3. Two-player version of the tragedy of the commons game, with the payoff matrix $[R, S, T, P]=\left[0,-\frac{c}{2}, b-\frac{c}{2}, b-c\right], b<c<2 b$, and $r>b$. For all these cases (which are particular cases of the general PD game) $[R, S, T, P]$ with $T>R>P>S$, we prove

Lemma 1 The stationary point $q=1$ of the replicator dynamics (9) is a global attractor for the $S V$ redistribution of the $P G$ game (11).

Proof For the game A1, the values of two-player coalitions are $v(\{C C\})=2 d, v(\{C D\})=$ $d+1, v(\{D D\})=2$. The redistribution procedure leads to the $\mathrm{SV}$ game (8), with $\left[R^{\prime}, F_{1}, F_{3}, F_{2}, F_{4}, P^{\prime}\right]=[r,(2 r+d / 2+1 / 2) / 3,(2 r-d+2) / 3,(r+d+1) / 3,(r-$ $d / 2+5 / 2) / 3,1]$. We obtain in (9), (10) $A=B=0, W(q) \equiv(r+d-2) / 3>0$, therefore $q=1$ is a global attractor in $(0,1]$.

2. For the games $\mathrm{A} 2$ and $\mathrm{A} 3$, the proof in analogous, we obtain (with normalization $c=1)$ in both cases $A=B=0$ and $W(q) \equiv(r+b-2) / 3>0$ for the game A2, $W(q) \equiv(r-b) / 3>0$ for the game A3; therefore, $q=1$ is a global attractor in $(0,1]$.

This result corresponds to the fact that $C$ strategy strongly dominates D strategy in the SV game (8), as can be easily checked. Thus, for the PG game from example 1a, redistribution of the total wealth reverses the stability of the points $q=0$ and $q=1$ : The point $q=1$ becomes a global attractor of the replicator dynamics, and the population evolves to full cooperation.

As we shall see, in examples $1 \mathrm{~b}$ and $1 \mathrm{c}$, redistribution fosters cooperation by squeezing the basin of attraction of the total defection $q=0$. In both games from examples $1 \mathrm{~b}$ and $1 \mathrm{c}$, as a complementary two-player game required to define the SV's of the players, we choose the two-player Stag Hunt game:

$$
\begin{array}{c|cc} 
& C & D \\
\hline C & d & 0 \\
D & 1 & 1
\end{array}
$$

where $d \in(1,2)$ denotes the payoff of each player if they both hunt the stag, $C$ stands for hunting the stag, $D$ for hunting the hare. The game can be also seen as a two-player PG game with the threshold $M=2$. We prove

Lemma 2 For the SV redistributions of the PG games (12) (Example 1b) and (13) (Example $1 \mathrm{c})$, there exists a unique stationary point $q^{*} \in(0,1)$ of the replicator equations $(10) . q^{*}$ is a repeller. In both cases, the basin of attraction of the equilibrium $q=0$, corresponding to full defection, shrinks after the redistribution procedure.

Proof Case 1b:

The SV game has the payoff matrix (8), with $\left[R^{\prime}, F_{1}, F_{3}, F_{2}, F_{4}, P^{\prime}\right]=[r,(2 r+d / 2+$ $1 / 2) / 3,(2 r-d+2) / 3,(r+d+1) / 3,(r-d / 2+5 / 2) / 3,1]$. From (9) and (10), we 
calculate $W(q):=-3 r q^{2}+2(2 r+d) q-2$, which has two positive roots $q_{1}<q_{2}$ : $q_{1,2}=\frac{2 r+d \mp \sqrt{(2 r+d)^{2}-6 r}}{3 r}$, for all $r, d>1$, with $q_{1} \in(0,1), q_{2}>1$. From the structure of the rhs of (9), we deduce that $q_{1}$ is a repeller.

We remind that for $r \leq 9 / 4$, the population with interactions described by the game $1 \mathrm{~b}$ without redistribution evolves to global defection, whereas in the same model with redistribution, the polymorphism is possible as the final state of the population.

For $r>9 / 4$, we compare $q_{1}$ with $q_{1}^{w}:=\frac{2}{3}-\frac{1}{2 r} \sqrt{\frac{16 r^{2}}{9}-4 r}$, the unstable equilibrium for the model without redistribution. Straightforward, but somewhat tedious calculations show that $q_{1}^{w}>q_{1}$ for all positive $r, d$, i.e., the basin of attraction of full defection decreases when redistribution is enforced.

Case 1c: The SV game has the payoff matrix (8), with $\left[R^{\prime}, F_{1}, F_{3}, F_{2}, F_{4}, P^{\prime}\right]=[r,(d+$ $1 / 2) / 3,1 / 3,2(1-d) / 3,5 / 6,1]$. From (9) and (10), we calculate $W(q)=r q^{2}+\frac{2}{3} d q-\frac{2}{3}$, which for all $r>0, d>1$ has an unique zero $q_{2}=-\frac{d}{3 r}+\frac{1}{r} \sqrt{d^{2} / 9+2 r / 3} \in(0,1)$, a repeller of (9). By straightforward calculation, we check that the unstable equilibrium $q_{0}:=\frac{1}{\sqrt{r}}$ of the game (13) and the unstable equilibrium $q_{2}$ of the redistributed game satisfy $q_{0}>q_{2}$. In this case, the enlarging of the bassin of attraction of the cooperative strategy could be expected, since already in the two-player Stag Hunt game (14), it is larger than that of the three-person Stag Hunt game (13).

Thus, in both cases $1 \mathrm{~b}$ and $1 \mathrm{c}$, the redistribution procedure reduces the basin of attraction of full defection, i.e., fosters cooperation in the long run.

To have a deeper insight into the conditions which foster cooperation, in "Appendix", we present analytical results for two-player games and their SV redistributions. In particular, we show that the SV redistribution of the general two-player PD game always fosters cooperation, and the SV redistributions of the general coordination and anti-coordination games foster cooperation if a simple additional condition, the same for both types of games, is satisfied.

In general, the choice of the values of smaller coalitions is not unique. The choice can be motivated by the properties of the underlining initial $n$-player game or by a particular social situation, in which lower order games could have different social tension build in. For example, in larger groups, players could be more inclined to display PD behavior, whereas interacting in pairs they would rather prefer less social tension.

Example 2 (three-player PD game) We present here the results for the general three-player PD game. To preserve the "social tension" pertinent to the "dilemma" of the PD game, we assume in (3)

$$
R^{\prime}>S^{\prime}>S^{\prime \prime}, \quad T^{\prime \prime}>T^{\prime}>P^{\prime}, \quad T^{\prime \prime}>R^{\prime}, \quad T^{\prime}>S^{\prime}, \quad P^{\prime}>S^{\prime \prime} .
$$

In particular, strategy D strictly dominates $C$; therefore, the replicator dynamics based on the PD game without redistribution gives $q=0$ (corresponding to $\mathrm{D}$ ) as the asymptotic equilibrium state. We performed extensive studies for the redistributed game for the payoffs vectors with integer coordinates (15) between 0 and 10 . The examples below are generic for all considered games. In examples below, $P_{2}=[R, S, T, P]$ denotes the payoff matrix (4), $P_{3}=$ $\left[R^{\prime}, S^{\prime}, S^{\prime \prime}, T^{\prime \prime}, T^{\prime}, P^{\prime}\right]$ stands for the payoff matrix (3), and $R_{3}=\left[R^{\prime}, F_{1}, F_{3}, F_{2}, F_{4}, P^{\prime}\right]$ denotes the redistributed payoff matrix (8). In the last two columns, $q_{i} \in(0,1)$ denote mixed equilibria (if they exist) of (9), unst stands for unstable, st for stable. Stability refers to local or global stability, depending on the context. 


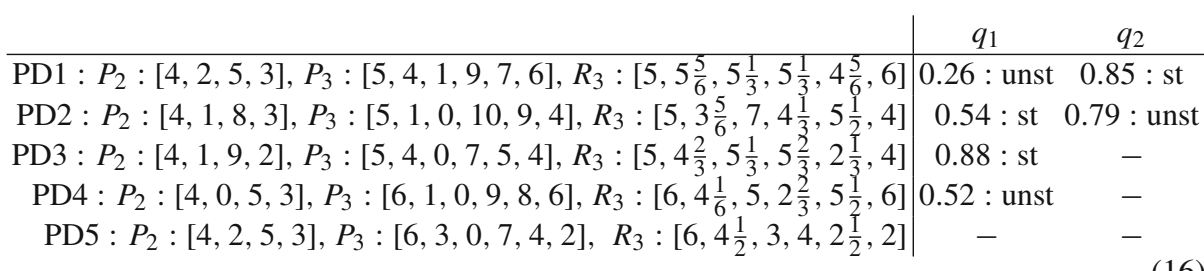

For example in the game PD1, the locally stable polymorphic equilibrium $q_{2} \approx 0.85$ is reached if the initial frequency of cooperators in the population is larger than $\approx 0.26$; otherwise, the system evolves to total defection. In PD5, all nonzero initial frequencies of cooperators lead to full cooperation. In this case, the matrix $R_{3}$ of the $\mathrm{SV}$ redistributed game reads

\begin{tabular}{c|ccc} 
& $C C$ & $C D$ & $D D$ \\
\hline$C$ & 6 & $4 \frac{1}{2}$ & 3 \\
$D$ & 4 & $2 \frac{1}{2}$ & 2
\end{tabular}

with strategy $C$ strongly dominating strategy $D$. In all other cases, there is no strong domination in the SV redistributed game. In all cases, the redistribution procedure fosters cooperation in the general three-player PD: Asymptotic partial or full cooperation is either granted for all nonzero initial frequencies of cooperators or is reached for the initial frequencies large enough. The total defection $q=0$ has never been found to be a global attractor.

There exist important classes of three-player PD games, for which the property: Strategy $D$ does not dominate strategy $C$ in the redistributed game, can be shown analytically. We prove

Lemma 3 If in the initial three-player game (3) the payoff of each player is a sum of payoffs from two PD games (4) played with both opponents and an auxiliary general two-player game (4) satisfies $R>P$, then in the redistributed game (8), strategy $D$ does not dominate strategy $C$.

Proof The condition: In (3), the payoffs are sums of two-player PD games (4), reads $R^{\prime}=$ $R, S^{\prime}=(R+S) / 2, S^{\prime \prime}=S, T^{\prime \prime}=T, T^{\prime}=(T+P) / 2, P^{\prime}=P$. Inserting these values in (6), (7) we obtain $F_{1}>F_{4}$.

Lemma 4 If the initial three-player game is the PD game in the benefit cost formulation, i.e., $P_{3}=\left[R^{\prime}, S^{\prime}, S^{\prime \prime}, T^{\prime \prime}, T^{\prime}, P^{\prime}\right]=[b-c, b / 2-c,-c, b, b / 2,0]$, or the $P G$ game from example 1a and an auxiliary two-player game (4) satisfies $R>P$, then in the redistributed games (8), strategy $D$ does not dominate strategy $C$.

Proof For the PD game, straightforward calculations give, from (6), (7), $F_{1}-F_{4}=b-c+$ $R-P>0$. For the $\mathrm{PG}$ game from example 1a, we obtain $F_{1}-F_{4}=r-1+R-P>0$.

The statement of both lemmas holds for all two-player games which satisfy $R>P$. In "Appendix", we show that if the initial two-player strategic game is a general PD game, then in redistributed, two-player game defection is never a dominant strategy. Thus, in the considered two-player and three-player PD games, redistribution produces games with cooperation as dominant or coexisting strategy. 


\section{Discussion}

In this exploratory study, we proposed a theoretical framework in which we have integrated the ideas of strategic and coalition games played in an evolutionary setting into one mathematical model. We proposed a particular method of redistribution of the value of a coalition: the $\mathrm{SV}$ redistribution. It will be of interest to investigate other methods of redistribution, the egalitarian one (with evenly divided total payoff of the coalition), and redistributions based on another solution concepts, e.g., on the so called "procedural values", which generalize the Shapley value, cf. [7], the nucleolus, the least core, or an imputation from the core. In the latter case, this could give rise to stochastic models, since the core is in general not a singleton in our models. More detailed and systematic study will be presented elsewhere.

In our model, the redistribution of the original payoffs from the strategic game played in the coalitions results in a different strategic game. Similar ideas, in different context, were already present in game theory, see e.g., [8] and references therein. The changes of the game types were discussed for the two-player PD games in a different setting. In $[11,12]$, the authors considered population models in which the agents seek new connections at different rates. In the limit in which the dynamics of the network is much faster than the evolution of strategies, the authors show that the PD game on such networks can be transformed to a coordination game and the Snow Drift game to a harmony game- the transformations change the rules of the game and explains the emergence of cooperation in the considered model.

It can also be of interest to generalize the proposed procedure to strategic and coalitional games on networks, cf. e.g., [15,19], in which the agents could enter different coalitions with their neighbors. The players could also take into account popularity of a strategy in the neighborhood, cf. [6] and references therein, where such idea was discussed for infinite populations.

The biological micro-systems with competing and cooperative relationships could also be investigated in the frame of similar evolutionary coalitional games, cf. [5]. Such games could possibly be applied to study cooperative and noncooperative behavior in economics [14].

The proposed general frame can be applied to any strategic game, in particular to the general public good game and to repeated games. In this respect, it will be of interest to study higher order multiplayer games, cf. e.g., [1,2]. Games with more than two strategies can be also studied in the proposed frame.

We have assumed that the population is divided into the coalitions of the same cardinality. This assumption can be weakened, assuming that the coalitions of various sizes are formed with prescribed probabilities, see also [3] where the PG games played in groups of different sizes were studied and [13] for a review on evolutionary dynamics of group interactions. In various coalitions (e.g., of different cardinality), different types of games can be played. For example, two-player interactions could have only fear build in, as described by a snow drift type game, whereas three-player interactions could have both fear and greed, as described by a three-player PD game.

Acknowledgments The author thanks two anonymous Referees for helpful comments and suggestions.

Open Access This article is distributed under the terms of the Creative Commons Attribution License which permits any use, distribution, and reproduction in any medium, provided the original author(s) and the source are credited. 


\section{Appendix}

We discuss the case $n=2$, i.e., a population in which two-player coalitions are formed. The initial strategic game (4) is SV transformed into the double symmetric game with the payoff matrix

\begin{tabular}{c|cc} 
& $C$ & $D$ \\
\hline$C$ & $R$ & $(T+S) / 2$ \\
$D$ & $(T+S) / 2$ & $P$
\end{tabular}

Note that for two-player coalitions, the SV redistribution is equivalent to the egalitarian redistribution, in which both players receive the same share of the total wealth of the coalition. We shall use the following abbreviations and definitions.

IG will stand for the initial game (4), SVG for the SV redistributed game (18), and RD for replicator dynamics.

Definition 1 IG is a coordination (anti-coordination) game if $R>T, P>S(R<T, P<$ $S$ ) and a harmony game if $R \geq T, S \geq P$.

SVG is a coordination (anti-coordination) game if $R>(T+S) / 2, P>(T+S) / 2$ $(R<(T+S) / 2, P<(T+S) / 2)$ and a harmony game if $R \geq(T+S) / 2 \geq P$.

Definition 2 We say that SVG fosters cooperation if one of the following conditions is satisfied:

1. in $\mathrm{RD} \dot{q}=q(1-q) W(q)$, derived from $\mathrm{SVG}, q=1$ is a global attractor in $(0,1]$,

2. the bassin of attraction of $q=1$ is larger than that for IG,

3. there exists $q_{\mathrm{SVG}} \in(0,1)$ - $\mathrm{a}$ global attractor of RD derived from SVG and $q_{I G} \in$ $[0,1)$ - a global attractor of RD derived from IG, such that $q_{\mathrm{SVG}}>q_{I G}$, i.e., the asymptotic level of cooperation in SVG is higher then in IG.

We prove

Lemma 5 If IG (4) is a PD game, then SVG fosters cooperation.

Proof If $(T+S) / 2>R$, then $\mathrm{SVG}$ is an anti-coordination game, i.e., there exists $q_{\mathrm{SVG}} \in$ $(0,1)$-repeller of the related RD, such that $\left(q_{\mathrm{SVG}}, 1\right]$ is the bassin of attraction of $q=1$. In the initial PD game, it was just the single point $q=1$. If $R \geq(T+S) / 2 \geq P$, then SVG is a harmony game. If $(T+S) / 2<P$, then SVG is a coordination game, with a point $q_{\mathrm{SVG}} \in(0,1)$-global attractor of the RD derived from SVG.

In consequence, in the evolutionary setting, after SV redistribution, the point $q=0$, corresponding to the population in which all players use strategy $\mathrm{D}$, is never a global attractor of the RD derived from $\mathrm{SVG}$, i.e., $\mathrm{SVG}$ is never a PD game.

We note that Lemma 5 holds with a more general sufficient condition $R \geq P$.

Below, we find conditions, sufficient to foster cooperation for other types of games. We define $M:=R+P-T-S$. For $M \neq 0$, we define the numbers (in Lemma 6 below they belong to $(0,1))$ :

$$
q_{I G}:=\frac{P-S}{M}, \quad q_{\mathrm{SVG}}:=\frac{P-\frac{T+S}{2}}{M} .
$$

We prove

Lemma 6 If $T>S$ and $I G$ is a coordination game or an anti-coordination game, then SVG fosters cooperation. 
Proof For a coordination IG, we have $R>T>\frac{T+S}{2}$.

If $P>\frac{T+S}{2}$, then SVG is also a coordination game, and, with $M>0, q_{I G} \in$ $(0,1), q_{\mathrm{SVG}} \in(0,1)$ are unstable equilibria of the relevant $\mathrm{RD}$, with $q_{\mathrm{SVG}}<q_{I G}$. Thus, the bassin of attraction of $q=1$ is larger for SVG.

If $P \leq \frac{T+S}{2}$, then $\mathrm{SVG}$ is a harmony game, which means that strategy $\mathrm{C}$ dominates strategy D, i.e., $q=1$ is a global attractor in $(0,1]$.

For an anti-coordination IG, we have $T>S>P, \frac{T+S}{2}>P$.

If $R<\frac{T+S}{2}$, then SVG is also an anti-coordination game, and, with $M<0, q_{I G} \in$ $(0,1), q_{\mathrm{SVG}} \in(0,1)$ are global attractors of the relevant $\mathrm{RD}$, with $q_{\mathrm{SVG}}>q_{I G}$.

If $R \geq \frac{T+S}{2}$, then SVG is a harmony game, i.e., $q=1$ is a global attractor in $(0,1]$.

The condition $T>S$ is important. With notation $[R, S, T, P]$ for the payoff matrix (4), for example, SVG of the coordination IG $[6,4,0,5]$ reads $[6,2,2,5]$, and the corresponding unstable mixed equilibria satisfy $q_{\mathrm{SVG}}=\frac{3}{7}>q_{I G}=\frac{1}{7}$. For the anti-coordination IG $[4,7,5,1]$, its SVG reads $[4,6,6,1]$, and the relevant stable mixed equilibria satisfy $q_{I G}=$ $\frac{6}{7}>q_{\mathrm{SVG}}=\frac{5}{7}$. In two SVG's generated by IG's $[1,4,0,5],[0,8,2,4]$, strategy D strongly dominates strategy $\mathrm{C}$. In all these examples, $T<S$.

Lemma 7 1. If IG is an anti-coordination game, then SVG is not a coordination game.

2. If IG is a coordination game, then the SVG is not an anti-coordination game.

Proof 1. If $R \geq P$ then $\frac{T+S}{2}>\frac{R+P}{2} \geq P$. If $R<P$, then $2 R<T+P, R<\frac{T+P}{2}<\frac{T+S}{2}$. 2. If $R \geq P$ then $\frac{T+S}{2}<\frac{R+P}{2} \leq R$. If $R<P$ then $\frac{T+S}{2}<\frac{R+P}{2}<P$.

As particular cases, we show below SVG's for three popular versions of a general twoplayer PD and for other two-person social dilemmas.

For the benefit cost PD: $[b-c,-c, b, 0], b>c>0$, its $\mathrm{SVG}$, after rescaling of all payoffs by $(b-c)$, is the harmony game $[1,1 / 2,1 / 2,0]$.

For the Public Good Game: $\left[r, \frac{r}{2}, \frac{r}{2}+1,1\right]$, where $r>1$ is the multiplier of the common pool, and each player may contribute a unit, its SVG is a harmony game with the payoff matrix $\left[r, \frac{r+1}{2}, \frac{r+1}{2}, 1\right]$.

For the weak PD game: [1, 0, $b, 0], b>1$, its SVG has the payoffs matrix $[1, b / 2, b / 2,0]$, and for $1<b \leq 2$ is a harmony game, for $b>2$ an anti-coordination game.

For the Stag Hunt game $[d, 0,1,1], d>1$, its SVG remains the coordination game $[d, 1 / 2,1 / 2,1]$, with the size of the bassin of attraction of strategy $\mathrm{D}$ twice smaller then in the initial game.

For the Snow Drift game $[b-c / 2, b-c, b, 0], b>c>0$, its SVG is a harmony game with the payoff matrix $[a, a, a, 0], a:=b-c / 2$.

\section{References}

1. Broom M, Cannings C, Vickers GT (1997) Multi-player matrix games. Bull Math Biol 59(1997):931-952

2. Bukowski M, Miękisz J (2004) Evolutionary and asymptotic stability in symmetric multi-player games. Int J Game Theory 33(1):41-54

3. Hauert C, Holmes M, Doebeli M (2006) Evolutionary games and population dynamics: maintenance of cooperation in public goods games. Proc R Soc B 273:2565-2570

4. Hofbauer J, Sigmund K (1998) Evolutionary games and population dynamics. University Press, Cambridge

5. Hummert S et al (2014) Evolutionary game theory: cells as players. Mol BioSyst 10:3044-3065

6. Kukla E, Płatkowski T (2013) Onset of limit cycles in population games with attractiveness driven strategy choice. Chaos Solitons Fractals 56:77-82 
7. Malawski M (2013) "Procedural" values for cooperative games. Int J Game Theory 42:305-324

8. Mogielski K, Płatkowski T (2009) A mechanism of dynamical interactions for two-person social dilemmas. J Theor Biol 260:145-150

9. Myerson RB (1997) Game theory., Analysis of ConflictHarvard University Press, Cambridge

10. Nowak MA (2006) Evolutionary dynamics., Exploring the Equations of LifeHarvard University Press, Belkin

11. Pachecho JM, Traulsen A, Nowak MA (2006a) Active linking in evolutionary games. J Theor Biol 243:437-443

12. Pachecho JM, Traulsen A, Nowak MA (2006b) Coevolution of strategy and structure in complex networks with dynamical linking. Phys Rev Lett 97:258103

13. Perc M, Gomez-Gardenes J, Szolnoki A, Floria LM, Moreno Y (2014) Evolutionary dynamics of group interactions on structured populations: a review. J R Soc Interface 10:20120997. doi:10.1098/rsif.2012. 0997

14. Rashedi N, Kebriaei H (2014) Cooperative and non-cooperative Nash solution for linear supply function equilibrium game. Appl Math Comput 244:794-808

15. Szabo G, Fath G (2007) Evolutionary games on graphs. Phys Rep 446(4-6):97-216

16. Nash JF (1950) Equilibrium points in n-person games. PNAS 36:48-49

17. Sandholm B (2010) Population games and evolutionary dynamics. MIT Press, Cambridge

18. Shapley LS (1953) A value for n-person games. In: Tucker AW, Kuhn HW (eds) Contributions to the theory of games II. Princeton University Press, Princeton, pp 307-312

19. Szabo G, Szonoki A (2012) Selfishness, fraternity and other-regarding preference in spatial evolutionary games. J Theor Biol 299:81-87

20. Taylor PD, Jonker L (1978) Evolutionarily stable strategies and game dynamics. Math Biosci 40:145-156

21. Weibull JW (1995) Evolutionary game theory. MIT Press, Cambridge 